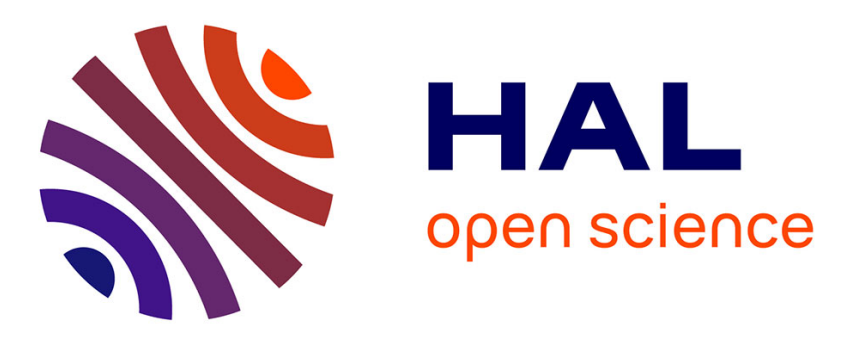

\title{
Analysis of Center of Mass and Gravity-Induced Vertebral Axial Torque on the Scoliotic Spine by Barycentremetry
}

Thomas Thenard, Claudio Vergari, Thibault Hernandez, Rapahaël Vialle, Wafa Skalli

\section{To cite this version:}

Thomas Thenard, Claudio Vergari, Thibault Hernandez, Rapahaël Vialle, Wafa Skalli. Analysis of Center of Mass and Gravity-Induced Vertebral Axial Torque on the Scoliotic Spine by Barycentremetry. Spine Deformity, 2019, 7 (4), pp.525-532. 10.1016/j.jspd.2018.11.007 . hal-02529577

\section{HAL Id: hal-02529577 https://hal.science/hal-02529577}

Submitted on 2 Apr 2020

HAL is a multi-disciplinary open access archive for the deposit and dissemination of scientific research documents, whether they are published or not. The documents may come from teaching and research institutions in France or abroad, or from public or private research centers.
L'archive ouverte pluridisciplinaire HAL, est destinée au dépôt et à la diffusion de documents scientifiques de niveau recherche, publiés ou non, émanant des établissements d'enseignement et de recherche français ou étrangers, des laboratoires publics ou privés. 


\title{
Analysis of Center of Mass and Gravity-Induced Vertebral Axial Torque on the Scoliotic Spine by Barycentremetry
}

\author{
Thomas Thenard, MSc ${ }^{\mathrm{a}, *}$, Claudio Vergari, $\mathrm{PhD}^{\mathrm{a}}$, Thibault Hernandez, MD ${ }^{\mathrm{a}, \mathrm{b}}$, \\ Raphael Vialle, $\mathrm{PhD}, \mathrm{MD}^{\mathrm{b}, \mathrm{c}}$, Wafa Skalli, $\mathrm{PhD}^{\mathrm{a}}$ \\ ${ }^{a}$ Arts et Metiers ParisTech, LBM/Institut de Biomecanique Humaine Georges Charpak, 151 bd de l'Hopital, 75013 Paris, France \\ ${ }^{\mathrm{b}}$ Service de Chirurgie Orthopédique et Réparatrice de l'Enfant, Hôpital Armand Trousseau, 26 avenue du Dr Netter, F-75012 Paris, France \\ ${ }^{\mathrm{c}}$ Département Hospitalo-Universitaire "Maladies Musculo-Squelettiques et Innovations Thérapeutiques" DHU-MAMUTH, Hôpital Armand Trousseau, 26 \\ avenue du Dr Netter, F-75012 Paris, France
}

\begin{abstract}
Study Design: Retrospective observational study.

Objectives: To determine the mass distribution along the scoliotic trunk using barycentremetry and its relationship with vertebral axial rotation and torque.

Summary of the Background Data: Deformity progression in adolescent idiopathic scoliosis (AIS) is not yet fully understood, but gravity load on the spine could play a role. Barycentremetry allows to characterize body mass distribution in standing position, which could provide a better understanding the mechanisms of progression.

Methods: 81 subjects (27 healthy adolescents and 53 AIS patients) underwent biplanar radiography and 3D reconstruction of the spine and body envelope. Position of the gravity line was estimated, as well as trunk segmental centers of mass COMs at each vertebral level and resulting axial torques to each vertebra.

Results: The COM of all trunk segments was less than $1 \mathrm{~cm}$ from the gravity line in the frontal plane for healthy subjects, and less than 1.5 $\mathrm{cm}$ for AIS patients. Vertebral axial torque was $0.7 \pm 0.5 \mathrm{Nm}$ in healthy subjects, $2.9 \pm 2.1 \mathrm{Nm}$ at the junctional vertebrae of AIS patients and $0.5 \pm 0.5$ at the apex. A strong association was found between high torque and high intervertebral rotation at junctions, with low torque and low intervertebral axial rotation at the apex.

Conclusion: Results suggest that AIS patients can maintain the COM of each body segment close to their gravity line, irrespective of the severity and asymmetry of their deformity. Moreover, torque analysis shed some light on the importance of junctional vertebrae in the spinal deformity and, potentially, in the vicious cycle determining scoliosis progression.
\end{abstract}

Level of Evidence: Level III.

Keywords: Adolescent idiopathic scoliosis; Center of mass; Axial torque; Intervertebral axial rotation

\section{Introduction}

Adolescent idiopathic scoliosis (AIS) is a 3D deformity of the trunk that can progress during growth spurt, potentially leading to postural imbalance or decreased lung

Author disclosures: TT (none), CV (none), TH (none), RV (grants from Eos Imaging, other from Spineguard, other from Stryker Spine, other from NuVasive, outside the submitted work), WS (coinventor of the EOS system, without personal financial benefit [royalties rewarded for nonprofit research and education institutions]).

IRB approval: Subject inclusion was approved by the ethical committee (6001 CPP Ile de France VI and local hospital ethical committee). capacity. The most common parameter used to characterize scoliosis severity is the angle of the curve (Cobb angle), which can be measured on frontal radiographs. However, given the $3 \mathrm{D}$ character of the deformity, transversal plane

Funding: The authors are grateful to the ParisTech BiomecAM chair program on subject-specific musculoskeletal modelling (with the support of ParisTech and Yves Cotrel Foundations, Société Générale, Proteor and Covea).

*Corresponding author. Institut de Biomécanique Humaine Georges Charpak, Arts et Métiers, 151 Boulevard de l'Hôpital, 75013 Paris, France. Tel.: +33 (0)1 442462 76; fax: +33 (0)1 44246366 .

E-mail address: thomas.thenard@ensam.eu (T. Thenard). 
geometry may have primary importance in the vicious cycle leading to progression [1,2], the mechanism of which is not yet fully understood.

The pioneering work of Duval Beaupère et al. has shown the relevance of spine and body balance when characterizing scoliosis [3]. More recently, the analysis of gravitational parameters such as the gravity line or the center of mass (COM) could be obtained from biplanar radiographs by estimating the mass distribution in different regions of the body to evaluate its segmental balance [4]. These estimations were obtained using force platform in standing position $[5,6]$. This approach allows estimating the gravity-induced loading of the spine at each vertebral level. Considering magnetic resonance imaging analysis, Adam et al. [7] estimated the overall torque applied to each vertebral level, whereas Keenan et al. [8] estimated vertebral torque in the coronal plane. However, the former study only roughly estimated the forces applied to the spine and the relative $\mathrm{COM}$ where forces were applied, whereas the latter was based on magnetic resonance imaging, with the patient in lying position.

Methods were proposed to obtain the patient's external envelope in standing position from biplanar radiographs [9]. It has been shown that with such methods, it is possible to accurately estimate the position of body COM both in healthy and scoliotic patients by estimating the density of each body segment $[4,10]$.

The hypothesis of the present work was that investigating body balance and the gravity-induced axial torque applied to the spine may increase our understanding of biomechanical aspects of scoliosis progression. The aim of this work was to estimate body balance and the axial torque applied to each vertebra for healthy subjects and scoliotic patients in standing position.

\section{Materials and Methods}

\section{Population}

Eighty adolescents were retrospectively included: 27 asymptomatic subjects (13 boys and 14 girls, mean age: $12.9 \pm 2.1$ ) and 53 AIS patients (13 boys and 40 girls, mean age: $14.4 \pm 1.7$, Cobb angle $31.6^{\circ} \pm 16.2^{\circ}$ ranging from $10^{\circ}$ to $76^{\circ}$ ) (Table 1 ). For AIS patients, junctional vertebrae and apical vertebra were selected by experienced surgeons based on radiographs. According to the SRS definition, the apical vertebra was defined as the most laterally shifted vertebra from the vertical axis in the frontal plane, which is also often the more axially rotated and horizontal. The junctional vertebrae, also called the inflexion vertebra, were defined as the vertebra where the curve changed direction from convex to concave and vice versa. In our study, we only focused on the junctional vertebrae from the major curve. The recruitment excluded patients with previous spine surgery, subnumerary, and supernumerary vertebrae. Subject inclusion was approved by the ethical committee (6001 CPP Ile de France VI and Trousseau hospital).

\section{$3 D$ reconstruction}

For each patient, head to feet low-dose biplanar radiographs were acquired using EOS system (EOS imaging, Paris, France). $3 \mathrm{D}$ reconstruction of the spine, the pelvis, and the external envelope (head, thorax, abdomen, arms, legs, and feet) was performed using validated techniques $[9,11,12]$ (Fig. 1).

Patient's gravity line (GL), vertebral axial rotations (VAR), and intervertebral axial rotations (IAR) were computed from the $3 \mathrm{D}$ reconstruction. The estimation of GL from 3D reconstruction of body envelope was validated on healthy and scoliotic patients [10], by comparison to a Wii Board, with a mean difference of $0.8 \mathrm{~kg}$ (SD 1.2) for the mass estimation and $0.5 \mathrm{~mm}$ (SD 1.2) for COM location, respectively.

\section{Barycentremetry}

The 3D envelope of each subject was cut into slices at each vertebral level, from T1 to L5, with horizontal planes passing through the center of each intervertebral disc (Fig. 2A), each slice being defined by a $\mathrm{COM}_{\text {slice }}$ (Fig. 2B). Each torso slice (between two cutting planes) contained the vertebra, the bottom half of the upper disc, and the top half of the lower disc. The head and neck were considered in a segment on its own. For each vertebra, the body segment above it (ie, the sum of the slices above it) was characterized by calculating its volume. Using the estimation of the density values proposed by Dempster [13] and by Amabile for the torso [14], its mass and center of mass ( $\left.\mathrm{COM}_{\text {segment }}\right)$ were also computed. The subject's arms were included in the model, although their position was not personalized, and their weight was shared by the T2 and T3 vertebrae.

\section{Calculation of the gravity-induced torque in the spine}

Calculation of the gravity-induced torque in each vertebra is illustrated in Figure 3. Axial torque, relative to the vertebra's vertical axis, depends on three parameters:

Table 1

Description of the different parameters of the population of patients, mean (SD).

\begin{tabular}{|c|c|c|c|c|c|c|c|c|}
\hline Population & Count & BMI & Age & Sex & Cobb angle $\left({ }^{\circ}\right)$ & T1-T12 kyphosis $\left({ }^{\circ}\right)$ & Lordosis $\left({ }^{\circ}\right)$ & Pelvic incidence $\left({ }^{\circ}\right)$ \\
\hline Asymptomatic & 27 & $18(1.97)$ & $12.9(2.1)$ & $\begin{array}{l}14 \mathrm{~F} \\
13 \mathrm{M}\end{array}$ & $X$ & $39.4(12.6)$ & $-44.1(9.9)$ & $48.4(11.1)$ \\
\hline Scoliotic & 53 & $19(2.59)$ & $14.4(1.7)$ & $\begin{array}{l}40 \mathrm{~F} \\
13 \mathrm{M}\end{array}$ & $31.6(16.2)$ & $36.6(12.7)$ & $-49.2(10.8)$ & $46.2(12.8)$ \\
\hline
\end{tabular}

F, female; BMI, body mass index; M, male; SD, standard deviation. 

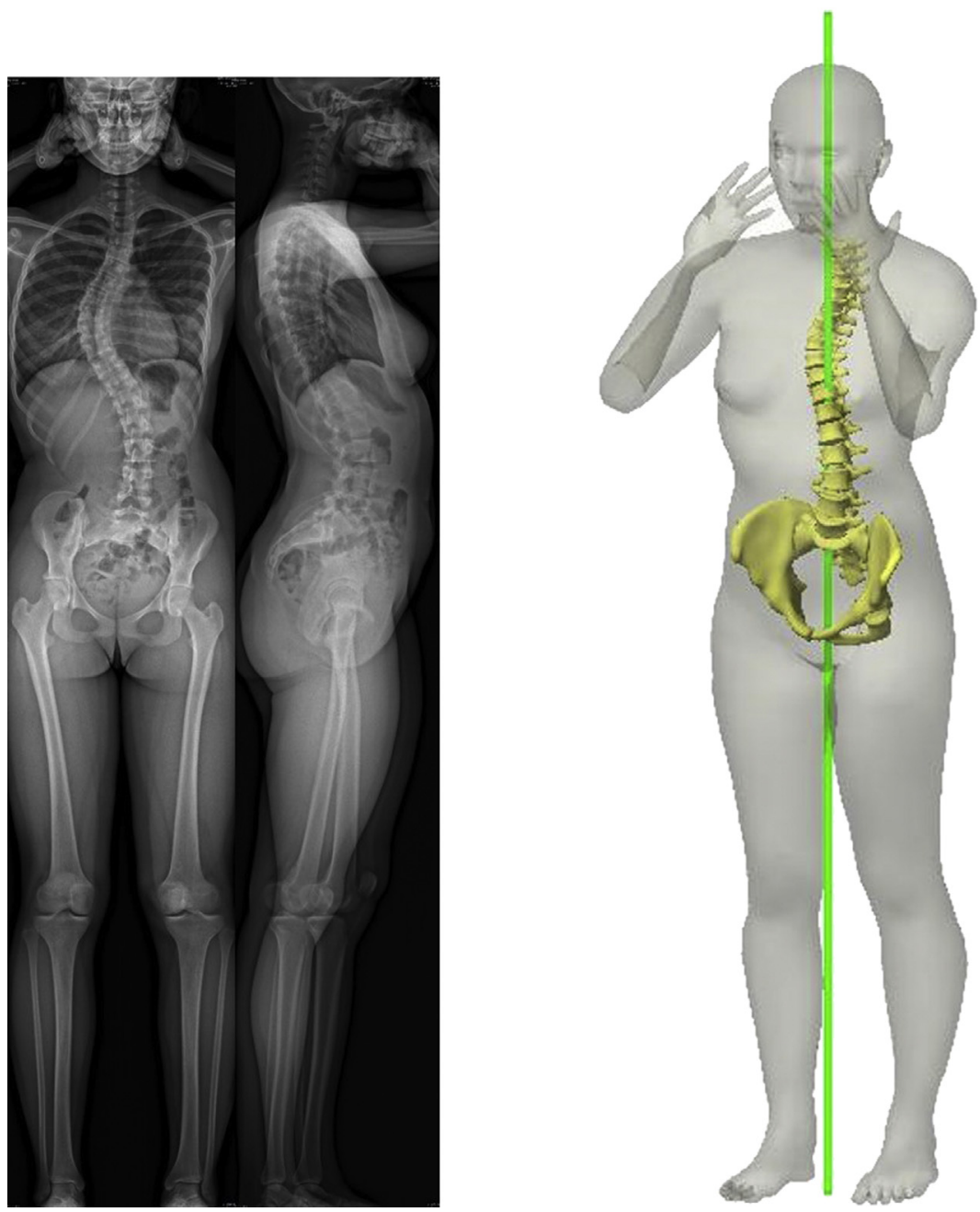

Fig. 1. Biplanar radiographs (left) and 3D reconstruction (right) of the external envelope and the spine, showering the gravity line passing through the center of mass.
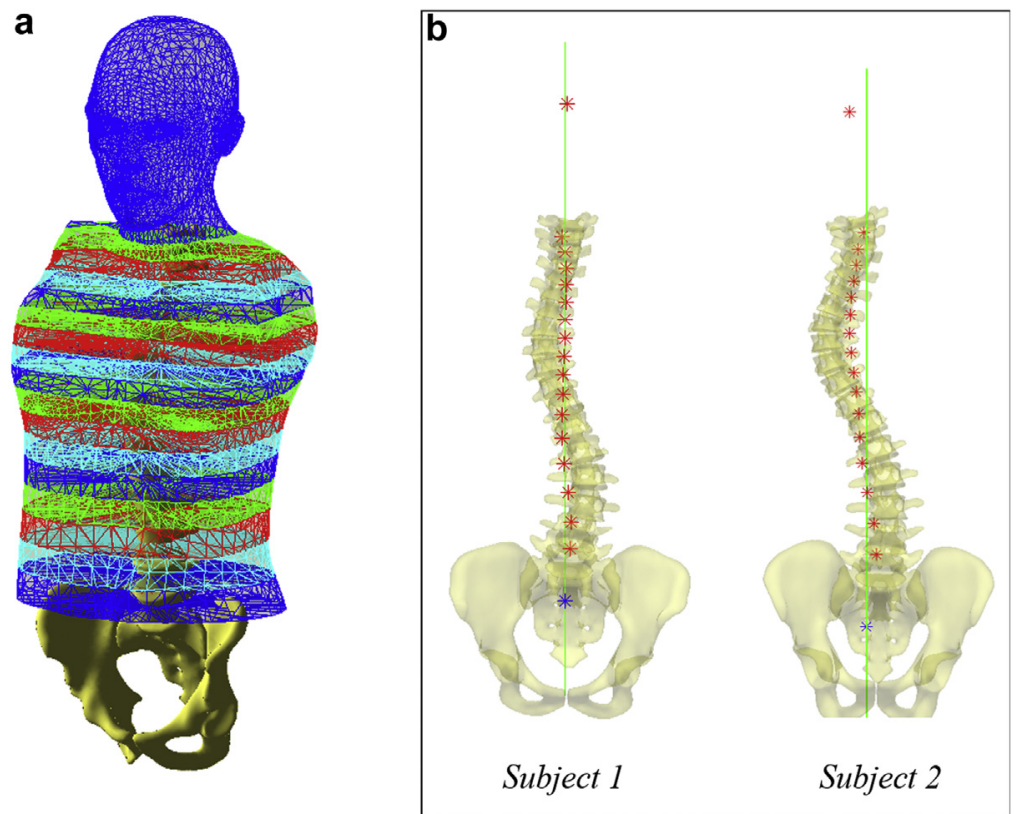

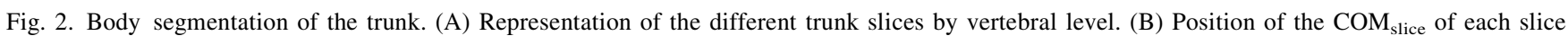
compared with the gravity line for two typical patients. 


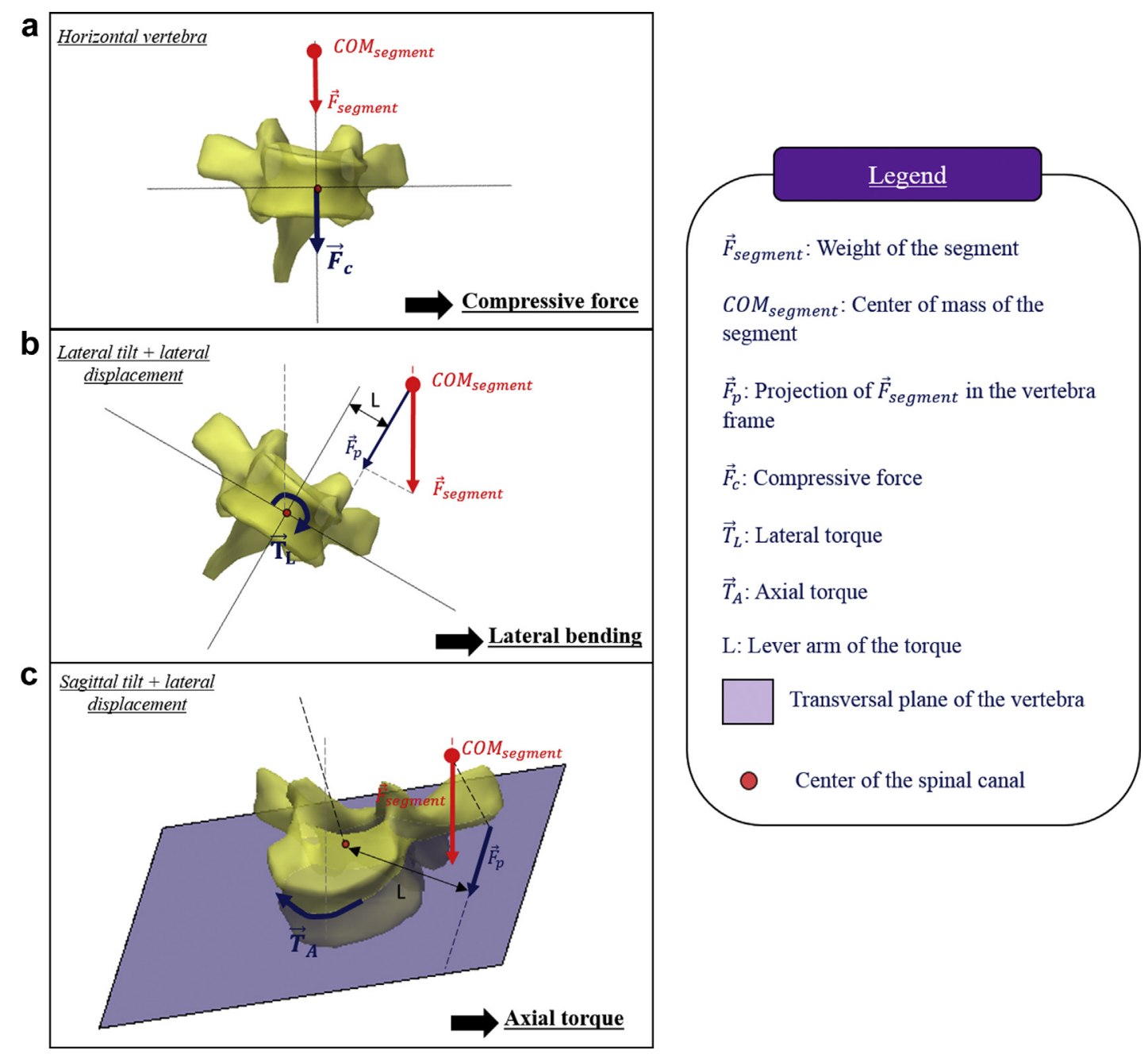

Fig. 3. Description of the different elements leading to the axial torque of a vertebra. (A) A horizontal vertebra is loaded with a vertical force that is aligned with the spinal canal. The weight of the segment above it will create a simple compressive force on the vertebra and no torque. (B) A vertebra is laterally tilted and laterally displaced relative to the $\mathrm{COM}_{\text {segment }}$. The lever arm L created by the lateral displacement of the vertebra and the projection of the weight Fp in the vertebral frame create a lateral bending of the vertebra. (C) A vertebra is tilted sagittally and displaced laterally. The lever arm L and the projection of the weight Fp tangential to the vertebra in the vertebral frame create an axial torque applied to the vertebra, producing the torsion.

the vertebra's orientation (axial, sagittal and lateral), the weight of the body segment above the vertebra, and the distance (ie, the moment arm) between the center of mass of this segment $\left(\mathrm{COM}_{\text {segment }}\right)$ and the spinal canal. In other words, torque is applied to a vertebra only if this vertebra is 1) laterally displaced relative to the center of mass and 2) tilted in the sagittal plane. The gravityinduced load applied at $\mathrm{COM}_{\text {segment }}$ for each vertebra was decomposed according to the vertebra's coordinate system [15]. The torque relative to the vertebral axis was defined as the cross product between the gravity-induced force $\mathrm{F}$ and the vector between $\mathrm{COM}_{\text {segment }}$ and the spinal canal (Fig. 3C). Absolute values of torque were considerated. Three examples are reported in Figure 3 to illustrate the phenomenon of axial torque. In the first case (Fig. 3A), a horizontal vertebra is loaded with a vertical force that is aligned with the spinal canal. In this case, the weight of the segment will create a simple compressive force on the vertebra and no torque. In the second case (Fig. 3B), a vertebra is laterally tilted and laterally displaced relative to the $\mathrm{COM}_{\text {segment }}$. The lever arm L created by the lateral displacement of the vertebra and the projection of the weight $\mathrm{Fp}$ in the vertebral frame create a lateral bending of the vertebra. In the third case (Fig. 3C), a vertebra is tilted sagittally and displaced laterally. The lever arm $\mathrm{L}$ and the projection of the weight Fp tangential to the vertebra in the vertebral frame create an axial torque applied to the vertebra, producing the torsion.

\section{Statistical analysis}

Normality corridors were defined from the asymptomatic population as the mean $\pm 2 \mathrm{SD}$ (95th centiles) for the lateral displacement of the COM, for the range of $\mathrm{COM}_{\text {segment }}$ position in the sagittal plane relative to the GL and for the vertebral axial torque. 
A

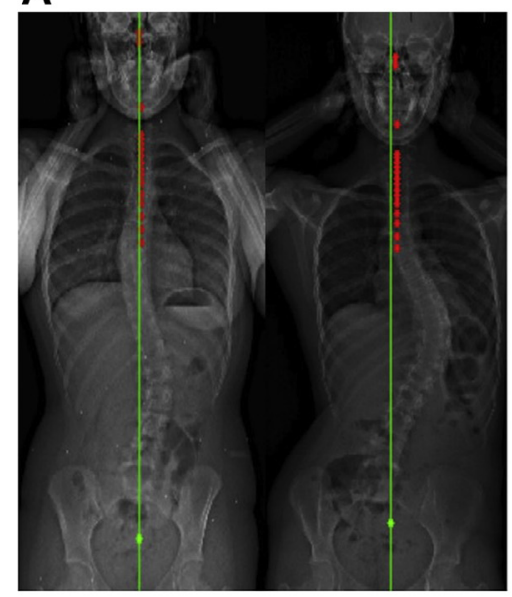

COM $M_{\text {segment }}($ red) compared to the GL (green) for a mild scoliotic patient (left) and a severe scoliotic patient (right).
B

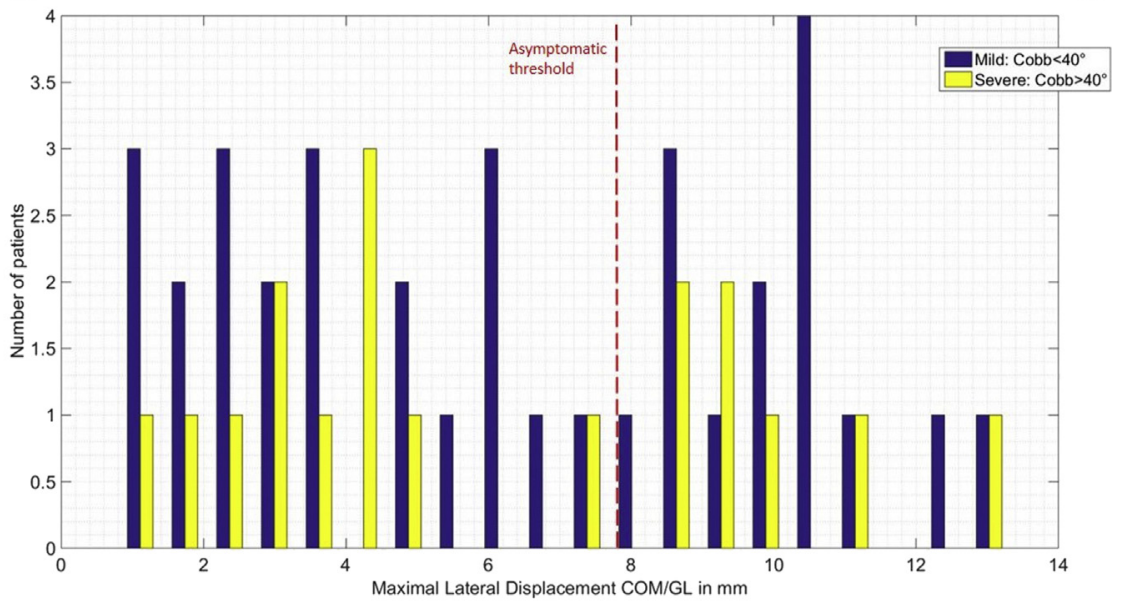

Fig. 4. Spatial distribution of the centers of mass of the segments above each vertebra for two representative AIS patients, one whose maximal distance was within the normality corridor (A) and one without (B). Vertical green lines represent the gravity lines. Panel C reports histograms of maximal lateral distance between the segmental centers of mass and the gravity line. The red dashed line represents the limit of the corridor of normality calculated on the asymptomatic population. Twenty-one patients were beyond this threshold, and there was no difference between mild and severe scoliosis.

Correlations were quantified using Spearman rank test. Subjects were analyzed by pathology group (scoliosis vs. healthy controls) with Mann-Whitney tests and by scoliosis topology (thoracic, thoraco-lumbar, lumbar) with KruskalWallis test. Torque and IAR values at junctions and apex of AIS patients were compared with the maximal values observed in each asymptomatic subjects.

The level of significance was set at 0.05. Data were analyzed using Matlab 2014b (The MathWorks Inc., Natick, MA).

\section{Results}

\section{Centers of mass}

Figure 2 shows two typical examples of the distribution of the $\mathrm{COM}_{\text {slice }}$ relative to the spine. $\mathrm{COM}_{\text {slice }}$ tended to follow the spinal line, but with much smaller displacement than the vertebrae. The maximal lateral distance between $\mathrm{COM}_{\text {segment }}$ and the GL for the healthy population gave a mean value of $3.9 \mathrm{~mm}$. The normality corridor (95th centile) was $8 \mathrm{~mm}$.

For the scoliotic group, the maximal lateral GL$\mathrm{COM}_{\text {segment }}$ distance gave a mean value of $6.2 \mathrm{~mm}$. Twenty-one patients of $53(39,6 \%)$ had maximal distances over the $8 \mathrm{~mm}$ threshold (Fig. 4). Comparison between the mild and severe scoliosis showed no differences $(p=.83)$, and no significant relationship was observed between the Cobb angle and this distance $(\mathrm{p}=.85)$.

\section{Gravity-induced torque}

Table 2 reports the values of torque, IAR, and axial orientations observed in the asymptomatic and AIS groups. AIS patients showed significantly higher torque and IAR than asymptomatic subjects at the junctional levels ( $\mathrm{p}<$ $.0001)$, and significant lower torque at the apex ( $p<$ $.0001)$. Indeed, 34 patients had maximal torque higher than the normality corridor, whereas 19 patients had higher IAR at the junctional levels.

Table 2

Vertebral axial torque and intervertebral axial rotation (IAR) and junctional and apical vertebrae.

\begin{tabular}{|c|c|c|c|c|}
\hline & Axial torque, $\mathrm{Nm}$ & & $\mathrm{IAR}^{\circ}{ }^{\circ}$ & \\
\hline Asymptomatic subjects $(\mathrm{n}=27)$ & Maximal value & & Maximal value & \\
\hline Mean \pm SD & $0.7 \pm 0.5$ & & $3.4 \pm 2.1$ & \\
\hline Range & $0-2.1$ & & $0-9.0$ & \\
\hline AIS patients $(\mathrm{n}=53)$ & Junctions & Apex & Junctions & Apex \\
\hline Mean \pm SD & $2.9 \pm 2.1$ & $0.5 \pm 0.5$ & $7.3 \pm 3.6$ & $0.98 \pm 0.8$ \\
\hline Range & $0.1-8.9$ & $0-1.8$ & $0.5-20$ & $0-3.2$ \\
\hline
\end{tabular}

AIS, adolescent idiopathic scoliosis; SD, standard deviation. 

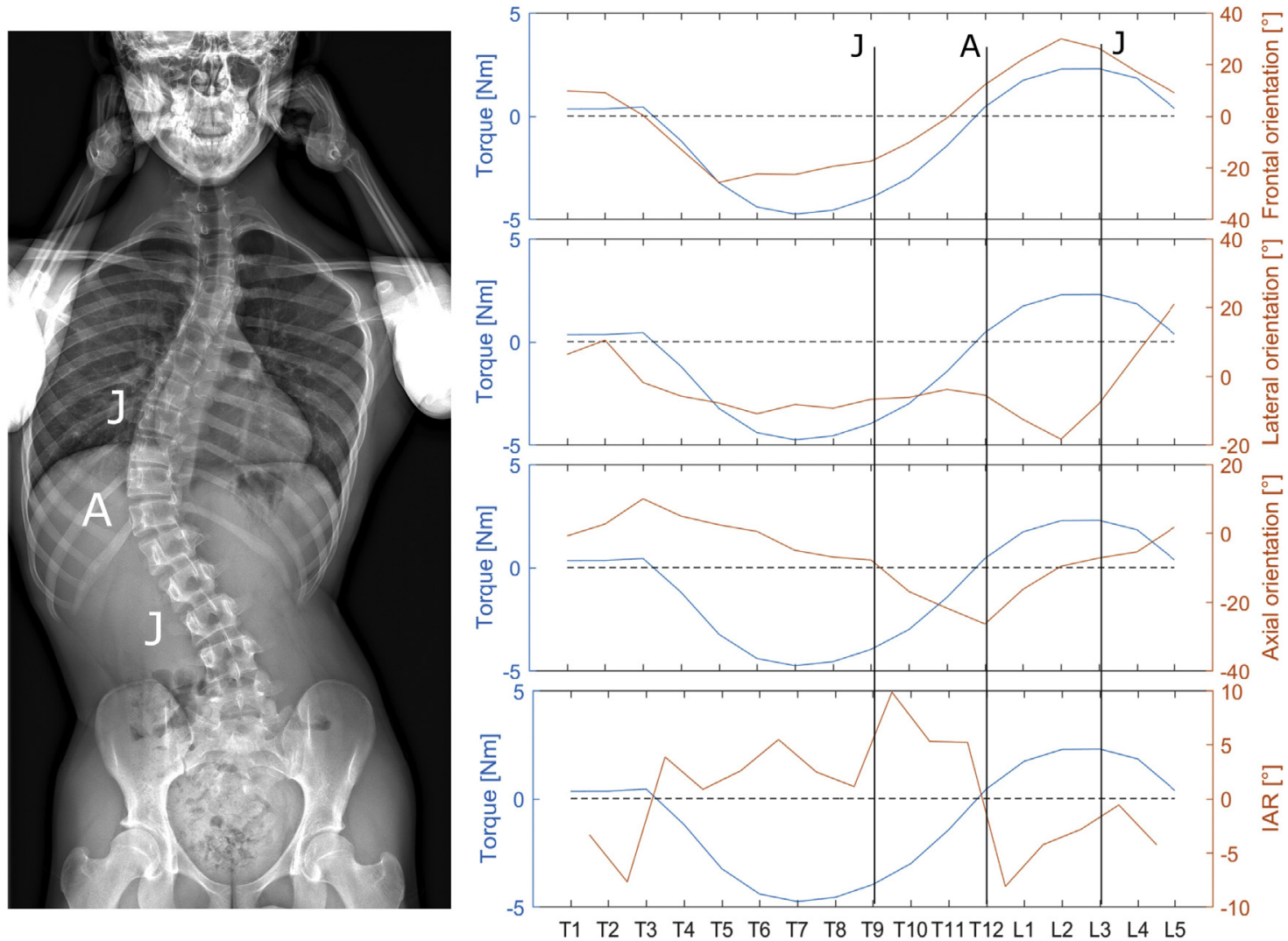

Fig. 5. Vertebral axial torque, vertebral orientation, and intervertebral axial rotation for a typical patient. Junctional (J) vertebrae and apex (A) are indicated. Axial orientation is maximal at the apex whereas intervertebral axial orientation is highest (and presents discontinuities) at the junctional levels. Torque is maximal near the junctionals and close to zero at the apex.

Figure 5 shows torque at all vertebral levels compared with the vertebral orientations and IARs for a typical scoliotic subject. VAR was high at the apex, whereas IAR and torque were higher near the junctions. Conversely, IAR and torque were minimal at the apex. This observation was confirmed for the whole AIS group, as shown in Figure 6. In the apical zone, axial torque was $30 \%$ of the torque at the higher junction and $49 \%$ of the lower junction (Fig. 6).

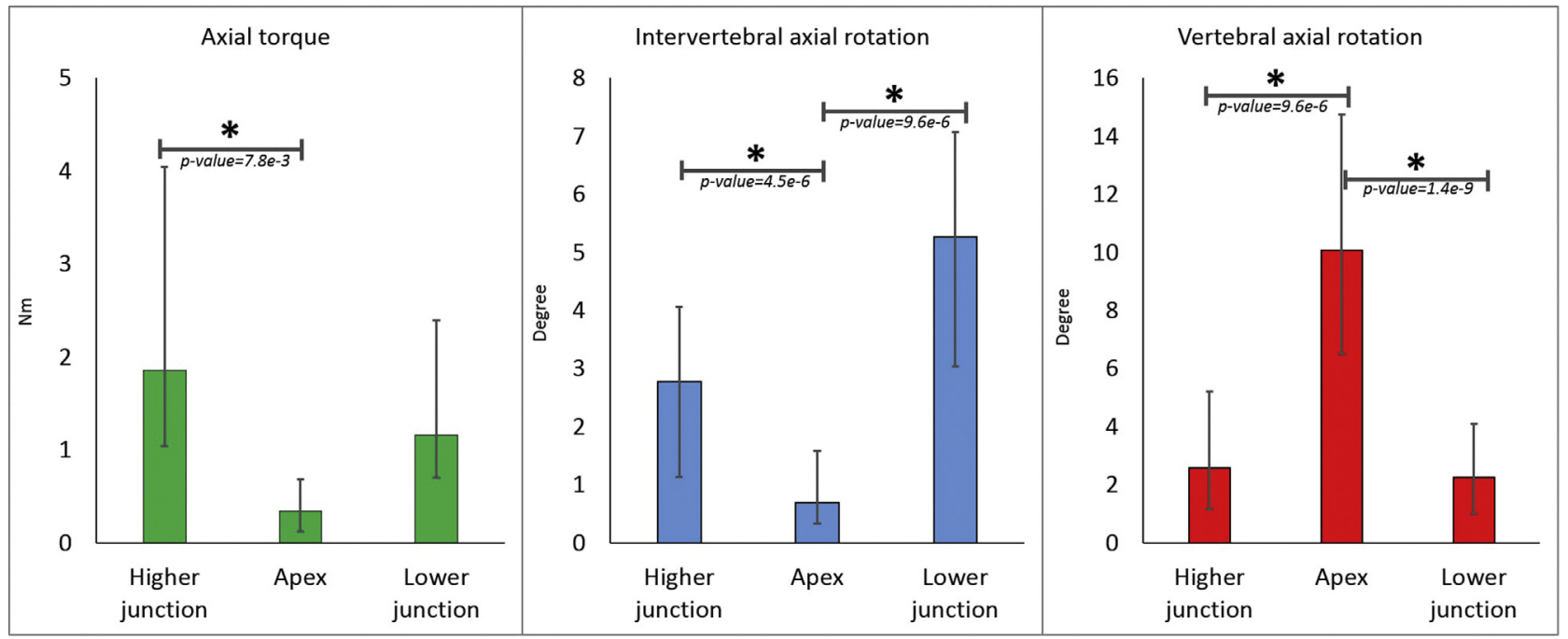

Fig. 6. Relation of the gravity-induced vertebral torque vertebral axial rotation (VAR), and intervertebral axial rotation (IAR), and the specific points of the major curve of scoliosis (apex, higher and lower junctional vertebra). Values are reported as median \pm first and third quartile. For the three parameters, significant differences were found between the value of the apex and the value of one of the junction. The asterisk shows a significant difference between two groups, p-values are indicated in the figure. 
A significant correlation was found between the axial torque at the junctions and the corresponding IAR $(\mathrm{p}<.05$, $r=0.65$ ), whereas no significant relationship was found for the healthy population. A significant correlation was also found between VAR along the spine and the distance between the vertebra and GL in the frontal plane, for more than $90 \%$ of the scoliotic population. In other words, vertebrae were more rotated the further they were from the gravity line.

\section{Discussion}

In this work, barycentremetry was used for a retrospective analysis of AIS patients in standing position, aiming at estimating their balance and the torsional forces applied to their vertebrae, in comparison with asymptomatic subjects.

The main limitation of this work is that the position of the arms was not personalized for each patient when reconstructing the body envelope. This could displace the gravity line and the position of the $\mathrm{COM}_{\text {segments. However, the body }}$ envelope model used is in the standardized free-standing position (Fig. 1), and patients who did not respect this position were excluded. This significantly limits the uncertainty of the gravity line position, as was confirmed by validation studies on healthy subjects and scoliotic patients, which reported an uncertainty of the GL position lower than $9 \mathrm{~mm}[10,14]$.

The second main limitation is that muscle action was neglected; therefore, only vertebral loading resulting from the gravity line was considered. The same approach was used previously in similar studies because of the complexity of load transfer to the spine. Musculoskeletal numerical modeling could be used to refine the analysis and to clarify the cause-effect relationship between torque, vertebral rotation, and IAR.

The analysis of the lateral displacement of the $\mathrm{COM}_{\text {segment }}$ compared with the gravity line gave an insight on the mass distribution and loading on the spine. The data reveals that the $\mathrm{COM}_{\text {segment }}$ of the asymptomatic population are well aligned with the gravity line, with a maximal lateral displacement lower than $1 \mathrm{~cm}$. For the scoliotic population, the lateral displacement of $\mathrm{COM}_{\text {segment }}$ did not exceed $1.5 \mathrm{~cm}$, even in cases of severe scoliosis. This distance is much lower than the lateral displacement of the single vertebra of the scoliotic spine. It can be concluded that the $\mathrm{COM}_{\text {segment }}$ of the mass supported by each vertebra remains almost aligned to the gravity line. Moreover, $\mathrm{COM}_{\text {segment }}$ position was not correlated to the magnitude of the spine deformation, represented by the Cobb angle. This suggests that scoliotic patients tend to remain globally well aligned, independently of the severity of the deformity.

Gravity-induced torque locally reached $8.5 \mathrm{Nm}$, with the higher values being at junctional levels (Fig. 6). It was recently suggested that high IAR could be characteristic of the geometric phenotype of progressive scoliosis [1], but the mechanical origin of high IAR remained unexplained. The present results suggest that IAR at junctions could be in relation with concentration of high torque at these levels, explaining to some extent the vicious cycle. On the other hand, $40 \%$ of the patients who presented abnormally high axial vertebral torque also had IAR at junctions within the normality corridor (Table 2). Disc ultrasonographic elastography of normal and scoliotic of intervertebral discs recently suggested that scoliotic discs may be stiffer than normal ones [16]; therefore, IAR could be a secondary factor.

In Figure 3, it can be noticed that if the vertebra was perfectly horizontal, the gravity load $\mathrm{F}$ would be parallel to the vertebra's vertical axis and it would generate no torque. Similarly, if the spinal canal was perfectly coincident relative to the weight of the trunk above it, there would be no lever arm and thus no torque. Adam et al. [7] also found an association between global spinal torque and intravertebral rotation, that is, the axial rotation between endplates. This end plate rotation (within the bone) is actually similar to IAR and, just like IAR, it is maximal at junctional levels. Because a nonzero vertebral torque requires an initial coronal deviation or torsion of the spine, those authors hypothesized that torque alone cannot explain the onset of the deformity. However, nonnegligible torques up to $2 \mathrm{Nm}$ were observed in the asymptomatic population in the present study. It is possible that such physiological albeit high torques could be sufficient to initiate a rotational deformity and therefore increase lateral scoliotic deviation.

Vertebral axial torque was the lowest at the apex (Figs. 5 and 6), which can be counterintuitive because the apex is the most laterally displaced vertebra of the curve. However, in this work, torque was calculated relative to the vertebra's vertical axis (Fig. 3), and therefore it strongly depends on the orientation of the vertebra. For instance, in the example shown in Figure 5, frontal orientation of the apex is almost zero (ie, T11 is horizontal in the patient's frontal plane), which means that there is no anteroposterior component to the gravity-induced loading of the vertebra, and therefore no axial torque. In this patient, torque continued increasing moving up from T11 until T4, even if the moment arms for those vertebrae were actually decreasing, indicating that vertebra orientation could be more important for axial torque than its distance from the gravity line. For this patient, torque closely follows frontal orientation; however, this was not the case for all patients.

Another important finding was the low torque at the apex, which is the vertebra that is most axially rotated. The same observation was done on the lateral bending moment: Keenan et al. [8] reported peak lateral bending moment at the apex level, which is often the most horizontal vertebra of the scoliotic curve. Indeed, the apex is the location of highest lateral bending moment and lowest axial torque, while at the same time being the most horizontal and most rotated vertebra.

\section{Conclusion}

Barycentremetry was used in this study to investigate patient balance and vertebral torsional loading. It appears 
that scoliotic patients tend to maintain their segmental centers of mass close to the gravity line at all vertebral levels, irrespectively of curve severity or clinical imbalance. Moreover, it was observed that vertebral axial torque is the highest at the junctional levels and the lowest at the apex. Although the underlying cause-effect mechanism still needs to be clarified, this could have an important impact on our understanding of the vicious cycle leading to progression of deformity, because it highlights the importance of the junctional vertebrae in the mechanics of the scoliotic spine.

\section{Acknowledgments}

The authors are grateful to the ParisTech BiomecAM chair program on subject-specific musculoskeletal modelling (with the support of ParisTech and Yves Cotrel Foundations, Société Générale, Proteor, and Covea).

\section{References}

[1] Skalli W, Vergari C, Ebermeyer E, et al. Early detection of progressive adolescent idiopathic scoliosis: a severity index. Spine 2017;42:823-30.

[2] Stokes IA, Spence H, Aronsson DD, Kilmer N. Mechanical modulation of vertebral body growth. Implications for scoliosis progression. Spine 1996;21:1162-7.

[3] Duval-Beaupère G, Robain G. Visualization on full spine radiographs of the anatomical connections of the centres of the segmental body mass supported by each vertebra and measured in vivo. Int Orthop 1987;11:261-9.

[4] Amabile C, Nérot A, Choisne J, et al. Alignment of centers of mass of body segments with the gravity line. Comput Methods Biomech Biomed Engin 2015;3:111.
[5] Schwab F, Lafage V, Boyce R, et al. Gravity line analysis in adult volunteers: age-related correlation with spinal parameters, pelvic parameters, and foot position. Spine 2006;31:E959-67.

[6] Steffen JS, Obeid I, Aurouer N, et al. 3D postural balance with regard to gravity line: an evaluation in the transversal plane on 93 patients and 23 asymptomatic volunteers. Eur Spine J 2010;19:760-7.

[7] Adam CJ, Askin GN, Pearcy MJ. Gravity-induced torque and intravertebral rotation in idiopathic scoliosis. Spine 2008;33:E30-7.

[8] Keenan BE, Pettet GJ, Izatt MT, et al. Gravity-induced coronal plane joint moments in adolescent idiopathic scoliosis. Scoliosis 2015;10:35.

[9] Nérot A, Choisne J, Amabile C, et al. A 3D reconstruction method of the body envelope from biplanar $\mathrm{x}$-rays: evaluation of its accuracy and reliability. J Biomech 2015;48:4322-6.

[10] Hernandez T, Vergari C, Thenard T, et al. Coronal trunk imbalance in idiopathic scoliosis: Does gravity line localisation confirm the physical findings? Rev Chir Orthop Traumatol 2018:442-8.

[11] Aubert B, Vergari C, Ilharreborde B, et al. 3D reconstruction of rib cage geometry from biplanar radiographs using a statistical parametric model approach. Comput Methods Biomech Biomed Eng Imaging Vis 2016;4:281-95.

[12] Humbert L, De Guise JA, Aubert B, et al. 3D reconstruction of the spine from biplanar X-rays using parametric models based on transversal and longitudinal inferences. Med Eng Phys 2009;31: $681-7$.

[13] Dempster WT. Space requirements of the seated operator: geometrical, kinematic, and mechanical aspects of the body with special reference to the limbs. Wright-Patterson Air Force Base. OH: Wright Air Development Center; 1955.

[14] Amabile C, Choisne J, Nérot A, et al. Determination of a new uniform thorax density representative of the living population from 3D external body shape modeling. J Biomech 2016;49:1162-9.

[15] Stokes IA. Three-dimensional terminology of spinal deformity. A report presented to the Scoliosis Research Society by the Scoliosis Research Society Working Group on 3-D terminology of spinal deformity. Spine 1994;19:236-48.

[16] Langlais T, Vergari C, Pietton R, et al. Shear-wave elastography can evaluate annulus fibrosus alteration in adolescent scoliosis. Eur Radiol 2018:28:2830-7. 